\title{
Introduction to Natural Fiber Reinforced Vinyl Ester and Vinyl Polymer Composites
}

\begin{abstract}
In recent years, the polymer industry has intensified its efforts to produce renewable material based polymer. Therefore, the use of natural fiber composites has been widely considered in various engineering sectors to replace conventional synthetic composite usage. This is because natural fiber composite properties are easily disposed and environmentally friendly in generating economic and sustainability societies. Vinyl polymer is a group of matrices comprising of thermosets and thermoplastics that are normally preferred as matrices with natural fibers. Both groups have their own unique features in benefiting their applications. The composite made of thermoset resin cannot be reprocessed or recycled. The composites of the thermoset matrix tend to provide good mechanical strength, are fragile and have low tensile effects. This is in contrast to the properties of the thermoplastic polymer that can be formed and diluted without changing its physical properties. Thermoplastic has excellent impact resistance and ductile. However, various approaches continue to be carried out by researchers to meet the requirements of natural fiber composites in different applications.
\end{abstract}

Keywords; Natural fiber; Yinyl polymer; Thermoset; Thermoplastic; Mechanical strength 